\title{
artigo
}

Soares, J.P.; Costas, S.E.J.; Nogueira, W.P.; Souza, L.T.; Lucena, C.K.R.; Pereira, I.L.; Oliveira e Silva, A.C.;

Aplicativos móveis de intervenção como estratégia de prevenção das infecções sexualmente transmissiveis: revisão integrativa

\section{Aplicativos móveis de intervenção como estratégia de prevenção das infecções sexualmente transmissiviseis: revisão integrativa}

Mobile intervention applications as a strategy to prevent sexually transmissible infections: integrative review Aplicaciones de intervención móvil como una estrategia para la prevención de infecciones sexualmente transmitidas: revisión integrativa

\begin{abstract}
RESUMO
Objetivo: identificar a produção científica acerca dos aplicativos móveis de intervenção como estratégia de prevenção das Infecções Sexualmente Transmissiveis (IST). Metodologia: Revisão Integrativa com busca nas bases CINHAL, PubMed, LILACS e Medline. Dos 13 artigos que compuseram a amostra, oito abordavam o uso de aplicativos móveis de intervenção e cinco de recrutamento de parceiros. Resultados: Majoritariamente os estudos mostraram que os usuários dos aplicativos móveis são jovens, homens que fazem sexo com homens, e que cursaram o ensino superior. Quanto à vulnerabilidade às IST/aids, possuem uma maior multiplicidade de parceiros e baixa adesão ao preservativo. Quanto ao uso dos aplicativos para prevenção das IST, os de intervenção mostraram ser eficientes, pois aumentaram o uso do preservativo e o conhecimento sobre IST nos países onde foram testados. Conclusão: Os aplicativos representam fator contribuinte para comportamentos de risco e IST, entretanto podem ser grandes aliados na prevenção.
\end{abstract}

DESCRITORES: Doenças Sexualmente Transmissiveis; Aplicativos Móveis; Vulnerabilidade; Prevenção.

\section{ABSTRACT}

Objective: to identify scientific production about mobile intervention applications as a strategy for the prevention of Sexually Transmitted Infections (STI). Methodology: Integrative Review with search in CINHAL, PubMed, LILACS and Medline databases. Of the 13 articles that comprised the sample, eight addressed the use of mobile intervention applications and five of partner recruitment. Results: Most studies have shown that users of mobile applications are young, men who have sex with men, and who have attended higher education. As for the vulnerability to STIs/AIDS, they have a greater multiplicity of partners and low adherence to condoms. As for the use of STI prevention applications, the intervention programs proved to be efficient, as they increased condom use and knowledge about STIs in the countries where they were tested. Conclusion: The applications represent a contributing factor for risk behaviors and STIs, however they can be great allies in prevention.

DESCRIPTORS: Sexually Transmitted Diseases; Mobile Applications; Vulnerability; Prevention.

\section{RESUMEN}

Objetivo: identificar la producción científica sobre aplicaciones de intervención móvil como estrategia para la prevención de infecciones de transmisión sexual (ITS). Metodología: Revisión Integrativa con búsqueda en las bases de datos CINHAL, PubMed, LILACS y Medline. De los 13 artículos que comprendieron la muestra, ocho abordaron el uso de aplicaciones de intervención móvil y cinco de reclutamiento de socios. Resultados: La mayoría de los estudios han demostrado que los usuarios de aplicaciones móviles son jóvenes, hombres que tienen sexo con hombres y que han asistido a la educación superior. En cuanto a la vulnerabilidad a las ITS/SIDA, tienen una mayor multiplicidad de parejas y poca adherencia a los condones. En cuanto al uso de aplicaciones de prevención de ITS, los programas de intervención demostraron ser eficientes, ya que aumentaron el uso del condón y el conocimiento sobre las ITS en los países donde fueron probados. Conclusión: las aplicaciones representan un factor que contribuye a los comportamientos de riesgo y las ITS, sin embargo, pueden ser grandes aliados en la prevención.

DESCRIPTORES: Enfermedades Sexualmente Transmisibles; Aplicaciones Móviles; Vulnerabilidad; Prevención.

RECEBIDO EM: 28/02/2020 APROVADO EM: 28/02/2020

\section{Juliana Pontes Soares}

Mestre em Enfermagem. Doutoranda em Saúde Coletiva na Universidade Federal do Rio Grande do Norte. Natal, Rio Grande do Norte, Brasil. https://orcid.org/000-0001-6203-9937 


\section{Sérgio Eduardo Jerônimo Costa}

Enfermeiro. Referência de Vigilância do Distrito Sanitário V, João Pessoa, Paraíba, Brasil. https://orcid.org/0000-0002-44393091

\section{Wynne Pereira Nogueira}

Enfermeira. Mestra em Enfermagem no Programa de Pós-Graduação em Enfermagem da Universidade Federal da Paraíba. João Pessoa, Paraíba, Brasil. https://orcid.org/000-0002-7492-7939

\section{Layane Trindade de Souza}

Enfermeira. Centro de Referência e Atendimento a Mulher. Patos, Paraíba, Brasil. https://orcid.org/000-0001-7124-2187

\section{Cintya Karina Rolim Lucena}

Enfermeira do Complexo Hospitalar Dr. Clementino Fraga, João Pessoa, Paraíba, Brasil. https://orcid.org/00000-0003-00966457

\section{Ivoneide Lucena Pereira}

Doutoranda em Enfermagem e Saúde na Universidade Federal da Paraíba. João Pessoa, Paraíba, Brasil. https:/orcid.org/0000003-1763-4635

\section{Ana Cristina de Oliveira e Silva}

Doutora. Professora adjunta do curso de Enfermagem da Universidade Federal da Paraíba. João Pessoa, Paraíba, Brasil. https://orcid.org/0000-0001-8605-5229

\section{INTRODUÇÃO}

A s Infecções Sexualmente Transmissíveis (IST) persistem como um problema de saúde pública em todo o mundo, e tanto a fácil disseminação quanto a subnotificação dos casos dificultam a quebra da cadeia de transmissão e o desenvolvimento de ações preventivas. Embora possuam outras vias de disseminação, a via sexual continua sendo a principal forma de transmissão das infecções ${ }^{(1)}$.

Estima-se que anualmente no mundo ocorram cerca de 357 milhões de novas IST curáveis, como a clamídia, gonorreia, sífilis e tricomoníase. Entre as IST incuráveis, o HIV está entre as mais prevalentes e, globalmente, no ano de 2017, havia 36,9 milhões de pessoas vivendo com HIV. Grande parte dos casos das IST está entre as populações vulneráveis: profissionais do sexo, homens que fazem sexo com homens, usuários de drogas injetáveis, presidiários, adolescentes e populações migrantes ${ }^{(2-4)}$.

Essas populações aderem a comportamentos de risco, tais como: multiplicidade de parceiros, uso inconsistente do preservativo, uso de álcool e outras drogas, e início precoce da atividade sexual, entretanto a autopercepção de risco entre alguns segmentos populacionais é baixa, não desper- tando o quanto estão vulneráveis às IST ${ }^{(5)}$.

Nesse contexto populacional e com o desenvolvimento tecnológico proporcionado pela globalização, tem modificado consideravelmente a forma de pessoas se conhecerem e se relacionarem. A variedade de sites e aplicativos disponíveis destinados à busca por parceiros aumentou o contato entre as pessoas de diferentes espaços geográficos, facilitando a busca por sexo e o aumento da disseminação de Infecções Sexualmente Transmissíveis (IST), principalmente entre a população mais jovem ${ }^{(6)}$.

Desse modo, pesquisadores têm desenvolvido aplicativos com a finalidade de informar sobre os métodos de prevenção das IST, saúde sexual, adesão à terapia antirretroviral, comportamentos de risco e testes de diagnóstico, visando reduzir a disseminação de infecções, conhecidos como aplicativos móveis de intervençãoo ${ }^{(7,8)}$.

Este estudo mostra-se de grande relevância para profissionais de saúde, populações vulneráveis, comunidade científica e sociedade, pois, compila informações importantes sobre a utilização dos aplicativos móveis e sua relação com as IST no que diz respeito à vulnerabilidade e prevenção.

Tendo em vista a importância de buscar informações sobre os aplicativos móveis no contexto das Infecções Sexual- mente Transmissíveis e considerando as diferentes percepções, a presente revisão integrativa teve como objetivo identificar a produção científica existente acerca dos aplicativos móveis de intervenção como estratégia de prevenção das Infecções Sexualmente Transmissíveis.

\section{METODOLOGIA}

Para se alcançar o objetivo proposto, realizou-se uma pesquisa descritiva do tipo revisão integrativa (RI), a qual possibilita uma análise da literatura de forma ampla e sistemática, além de analisar e sintetizar o conhecimento científico a respeito do objeto do estudo, por meio dos resultados de pesquisas anteriores, assim como, identificar lacunas que devem ser preenchidas mediante a realização de novos estudos ${ }^{(9)}$.

A presente revisão integrativa seguiu o cumprimento de etapas essenciais para seu desenvolvimento: identificação do tema e seleção da questão de pesquisa; estabelecimento dos critérios de elegibilidade; identificação dos estudos nas bases científicas; avaliação dos estudos selecionados e análise crítica; categorização dos estudos; avaliação e interpretação dos resultados e apresentação dos dados na estrutura da revisão integrativa ${ }^{(9)}$. 


\section{artigo}

Soares, J.P.; Costas, S.E.J.; Nogueira, W.P.; Souza, L.T.; Lucena, C.K.R.; Pereira, I.L.; Oliveira e Silva, A.C.;

Aplicativos móveis de intervenção como estratégia de prevenção das infecções sexualmente transmissiveis: revisão integrativa

Refletiu-se sobre a seguinte questão norteadora: Quais as evidências científicas sobre aplicativos móveis de intervenção na prevenção de Infecções Sexualmente Transmissíveis no período de 2008 a 2018?

Diante da crescente evolução e desenvolvimento das mídias sociais, os aplicativos móveis invariavelmente se modificam com o tempo. Desse modo, a busca por estudos recentes que envolvam a temática torna-se imperativa. Destaca-se que estudos envolvendo aplicativos de intervenção com a finalidade de prevenir e promover saúde foram datados dos últimos dez anos, período elegível para esta revisão.

Para compor o corpus da pesquisa buscaram-se artigos na Internet no período de agosto a novembro de 2018. Com o objetivo de evitar viés na seleção dos artigos, durante a fase de busca e seleção dos mesmos, dois pesquisadores atuaram de forma independente ${ }^{(10)}$.

A busca na literatura dos estudos primários foi realizada via Internet, nas seguintes bases de dados: PubMed, CI-
NAHL, LILACS E Medline. Para assegurar ampla e criteriosa busca, os descritores controlados e palavras-chave foram delimitados no Thesaurus de acordo com cada base de dados, ou seja, Medical Subject Headings (Mesh) e Descritores em Ciências da Saúde (DeCS). Utilizou-se os indicadores booleanos AND e OR. Para a busca na base de dados LILACS, além dos operadores booleanos foram utilizados os qualificadores, conforme apresentado no quadro a seguir (Quadro 1).

Quadro 1. Estratégias de busca nas bases de dados PubMed, CINAHL, LILACS e Medline. João Pessoa, PB, Brasil, 2018.

\begin{tabular}{|c|c|c|}
\hline BASES DE DADOS & ESTRATÉGIA DE BUSCA & $\begin{array}{l}\text { PUBLICAÇÕES } \\
\text { IDENTIFICADAS }\end{array}$ \\
\hline Pubmed & $\begin{array}{l}\text { sexually transmitted disease or sexually transmitted diseases AND mobile applica-tion } \\
\text { or applications, mobile or Apps, Mobile AND prevention or prevention and control [Mesh] }\end{array}$ & 42 \\
\hline CINAHL & $\begin{array}{l}\text { sexually transmitted disease or sexually transmitted diseases AND mobile applica-tion or } \\
\text { applications, mobile or Apps, Mobile AND prevention or prevention and control [Mesh] }\end{array}$ & 27 \\
\hline LILACS & $\begin{array}{l}\text { doença sexualmente transmissível/prevenção e controle OU doença de transmis-são se- } \\
\text { xual/prevenção e controle OU infecções sexualmente transmissí-veis/prevenção e controle } \\
\text { AND aplicativos em dispositivos móveis OU aplicativos móveis OU aplicativos para disposi- } \\
\text { tivos móveis [DeCS] }\end{array}$ & 26 \\
\hline Medline & $\begin{array}{l}\text { sexual infections AND mobile apps OR mobile applications OR apps [ key word] sexu- } \\
\text { ally transmitted disease or sexually transmitted diseases AND mobile applica-tion or } \\
\text { applications,mobile or Apps, Mobile AND prevention or prevention and control [Mesh] }\end{array}$ & 08 \\
\hline
\end{tabular}

Figura 1. Diagrama dos artigos selecionados para a Revisão Integrativa. João Pessoa, PB, Brasil, 2018.

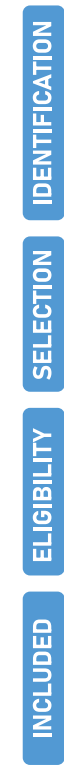

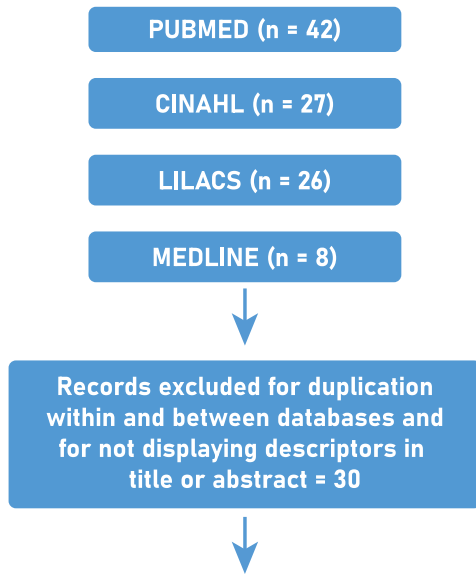

Full texts evaluated for eligibility $=73$

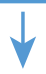

Studies included in the review $=13$
Full texts excluded for not being related to the theme $=60$
Para selecionar a amostra, foram utilizados os seguintes critérios de inclusão: artigos de pesquisa primária, indexados nas bases de dados selecionadas para o estudo e publicados no período de 2008 a 2018, que abordassem a temática nos idiomas português, inglês ou espanhol, disponíveis na íntegra. Os critérios de exclusão foram: ser artigo de revisão e duplicidade dentro e nas bases utilizadas e não apresentar os descritores no título ou no resumo dos artigos selecionados. Ressalta-se que os estudos duplicados foram considerados apenas uma vez.

Foram localizados 103 artigos, dos quais 30 foram excluídos por duplicidade dentro e entre as bases CINHAL, LILACS, PubMed, Medline e por não apresentar os descritores no título ou no resumo. Após a leitura do texto completo de 73 artigos, foram excluídos 60 por não ter relação com a temática do estudo. Desta forma, o corpus da presente revisão foi composto por 13 artigos, conforme a Figura 1. 
Os artigos selecionados foram sistematizados em um instrumento de coleta de dados, adaptado de um instrumento já validado $^{(10)}$, que permitiu a obtenção de informações sobre título, periódico e ano de publicação, objetivos, amostra, método e resultados. O processo de análise envolveu tradução e leitura dos artigos, e o preenchimento do instrumento com todos os dados do artigo. Os dados foram analisados tendo como base a relação com o objeto de estudo.

\section{RESULTADOS}

Com relação aos estudos selecionados, 2015 foi o ano com o maior número de publicações, totalizando cinco artigos, seguido por 2014 com três publicações, e os anos de 2012, 2013, 2016, 2017 e 2018 com uma publicação a cada ano. No período de 2006 a 2011 não se verificou estudos abordando a temática proposta.
Quanto ao tipo de delineamento de pesquisa dos artigos avaliados, evidenciou-se, na amostra: oito estudos transversais, dois estudos randomizados, três estudos de análise qualitativa (análise de conteúdo). Dessa forma em relação a força das evidências obtidas nos artigos, encontrou-se, onze artigos nível VI e dois artigos nível II ${ }^{(11)}$.

A observação do nível de evidência de cada estudo é importante para estabelecer a fidedignidade dos resultados que serão utilizados e, dessa maneira, fortalecer as conclusões que advirão da pesquisa em desenvolvimento. Os estudos podem ser classificados em sete níveis de evidência, a saber: Nível I - revisão sistemática ou meta-análise, proveniente de todos os ensaios clínicos controlados randomizados relevantes; Nível II - ensaio clínico randomizado; Nível III - ensaio clínico não randomizado; Nível IV - estudos de coorte ou de caso-controle;
Nível V - revisão sistemática de estudos descritivos e qualitativos; Nível VI - estudo descritivo ou qualitativo e Nível VII - estudos cuja evidência seja proveniente de opinião de autoridades e/ou relatório de comitês de especialistas ${ }^{(11)}$.

Os dados mostram a distribuição desses artigos em seis periódicos, e dentre eles destaca-se Aids and Behavior com cinco publicações nos anos de 2012, 2014, 2015, 2016 e 2017.

A maioria dos estudos selecionados para a presente revisão integrativa traz como população alvo os homens que fazem sexo com homens (HSH) e sua relação com o uso dos aplicativos móveis para busca por parceiros sexuais. Foram identificados oito que tratavam do uso dos aplicativos móveis de intervenção para a prevenção de IST e cinco que tratavam do uso de aplicativos móveis para recrutamento de parceiros, conforme Quadro 2 e Quadro 3.

Quadro 2. Quadro-síntese dos estudos relacionados aos aplicativos móveis de intervenção para a prevenção de Infecções Sexualmente Transmissivveis. João Pessoa, PB, Brasil, 2018.

\begin{tabular}{|c|c|c|}
\hline $\begin{array}{c}\text { TÍTULO, PERIÓDICO E ANO DE } \\
\text { PUBLICA-ÇÃO }\end{array}$ & OBJETIVOS & RESULTADOS \\
\hline $\begin{array}{l}\text { Putting Prevention in Their } \\
\text { Pockets: Developing Mobile } \\
\text { Phone-Based HIV Interventions } \\
\text { for Black Men Who Have Sex } \\
\text { with Men. } \\
\text { AIDS Patient Care and STDs, } 2013 .\end{array}$ & $\begin{array}{l}\text { Divulgar intervenções de saúde sobre Infec- } \\
\text { ções Sexualmente Transmissí-veis para Ho- } \\
\text { mens que fazem sexo com Homens (HSH) } \\
\text { negros através de aplicativos móveis. }\end{array}$ & $\begin{array}{l}\text { A tecnologia foi bem aceita como um meio de inter- } \\
\text { venção para prevenção no que diz res-peito ao HIV e } \\
\text { demais IST. }\end{array}$ \\
\hline $\begin{array}{l}\text { Online Outreach Services } \\
\text { Among Men Who Use the } \\
\text { Internet to Seek Sex with Other } \\
\text { Men (MISM) in On-tario, Canada: } \\
\text { An Online Survey. } \\
\text { Journal of Medical Internet Re- } \\
\text { search, 2015. }\end{array}$ & $\begin{array}{l}\text { Investigar as experiências de homens que } \\
\text { usam a internet para procurar sexo com ou- } \\
\text { tros homens e a autopercepção dos impactos } \\
\text { de divulgação de prevenção online. }\end{array}$ & $\begin{array}{l}\text { A maioria relatou que o prestador de serviços era } \\
\text { de difícil entendimento, porém útil. Em relação ao } \\
\text { comportamento, }(32,7 \%) \text { relataram o uso do pre- } \\
\text { servativo com maior frequência, realização de teste } \\
\text { para HIV }(34,4 \%) \text { ou IST }(28,6 \%) \text {, e }(25,2 \%) \text { buscaram } \\
\text { informações sobre o estado sorológico do parceiro. } \\
\text { Cerca de }(10,2 \%) \text { não relataram mudanças e a maio- } \\
\text {-ria }(83,8) \text { afirmou que usará o serviço nova-men- } \\
\text { te. Os que não utilizaram a divulgação de prevenção } \\
\text { online relataram não precisar desses serviços ou } \\
\text { não ter conhecimento sobre eles. }\end{array}$ \\
\hline $\begin{array}{l}\text { Prevention of Sexually Transmit- } \\
\text { ted Infections using mobile devi- } \\
\text { ces and ubiquitous computing. } \\
\text { International Journal of Health } \\
\text { Ge-ographic, } 2015 \text {. }\end{array}$ & $\begin{array}{l}\text { Investigar a contribuição da tecnologia para a } \\
\text { prevenção de Infecções Sexualmente Trans- } \\
\text { missí-veis. }\end{array}$ & $\begin{array}{l}\text { O software envia notificações preventivas aos utiliza- } \\
\text { dores quando detecta situações como a ativação de } \\
\text { aplicações específicas em seus smartphones, ou sua } \\
\text { proximidade a áreas com uma elevada probabilidade } \\
\text { de relação sexual (zonas quentes). As mensagens } \\
\text { utili-zadas foram selecionadas a partir de uma lista } \\
\text { que foi avaliada pelos próprios usuários. A mensagem } \\
\text { mais utilizada foi "Desfrutar do sexo e aproveitar a } \\
\text { vida. Não se exponha ao HIV". }\end{array}$ \\
\hline
\end{tabular}




\section{artigo}

Soares, J.P.; Costas, S.E.J.; Nogueira, W.P.; Souza, L.T.; Lucena, C.K.R.; Pereira, I.L.; Oliveira e Silva, A.C.

Aplicativos móveis de intervenção como estratégia de prevenção das infecções sexualmente transmissiveis: revisão integrativa

Acceptability and feasibility of using established geosocial and sexual networking mobile applications to promote HIV and STD testing among men who have sex with men.

AIDS Behavior, 2015.

Acceptability of Smartphone Appli-cation-Based HIV Prevention Among Young Men Who Have Sex With Men.

AIDS Behavior, 2014
Avaliar a aceitabilidade e viabilidade da prestação de informações sobre saúde sexual e referências de teste de HIV/DST esta-belecida via geosocial e aplicativos de redes se-xuais para homens que fazem sexo com homens (HSH).

Investigar a aceitabilidade de aplicativos de smar-tphone utilizados com a finalidade de prevenir o HIV.
MHealth approach to promote Oral HIV self-testing among men who have sex with men in China: a quali-tative description. BMC Public Health, 2018.
Explorar as opiniões dos Homens que fazem sexo com Homens na China sobre a viabilidade e potenciais preocupações de usar o WeChat para apoiar o HIVST e reduzir o risco de infecção pelo HIV.
Dois terços (64\%) dos aplicativos encontra-dos foram uma fonte aceitável para informa-ções sobre saúde sexual. Os HSH que aceita-ram não eram homens brancos, não tinham certeza do seu estado sorológico para HIV. Um quarto $(26 \%)$ dos bate-papos informativos de educação em saúde resultou na solicitação dos usuários nos referidos sites locais em testes de DST e HIV. Houve diferenças signifi-cativas no número e tipos de interações entre aplicativos.

A maioria dos participantes utilizava aplicati-vos para busca por parceiros sexuais e mani-festaram o desejo de participar de programas de prevenção de HIV através de aplicativos.

Os Homens que fazem sexo com homens descreveram o uso do WeChat e expressaram apoio ao uso desta plataforma para promover o HIVST e disseminar informações relacionadas ao HIV. Eles descreveram suas preferências sobre a implementação de uma intervenção de saúde móvel para promover o HIVST, incluindo a fonte de mensagens de intervenção, bem como tempo de mensagem, frequência, forma, tom e conteúdo. Os partici-pantes também descreveram preocupações relacionadas à privacidade sobre como rece-ber mensagens via WeChat e oferecer solu-ções potenciais.

Acceptability of HIV prevention information delivered through es-tablished geosocial networking mobile applications to men who have sex with men. AIDS Behavior, 2017

Avaliar o tipo de informa-ção sobre o HIV que os Homens que fazem sexo com homens estavam dispostos a receber atra-vés dos aplicativos esta-belecidos, bem como o método e frequência mais aceitáveis.

Using the Information-Motivation-Behavioral Skills Model to Guide the Development of an HIV Prevention Smartphone Application for High-Risk MSM. AIDS Educ Prev., 2015
Identificar o conteúdo desejado, recursos e funções de um aplicativo móvel para prevenção do HIV em homens que fazem sexo com homens de alto risco.
Todos os tipos de informações foram consi-derados aceitáveis e informações sobre pre-venção do HIV nos aplicativos usuais são necessárias.

Os participantes identificaram necessidade de informações relacionadas a prevenção do HIV tais como: centro de distribuição de testes e profilaxia do HIV; grupos de apoio; informa-ções sobre a doença e tratamento. Ressaltaram a importância de abordar nos aplicativos: uso correto do preservativo; negociação de sexo seguro; reconhecimento de sinais e sintomas de HIV e Infecções Se-xualmente Transmissiveis.

Quadro 3. Quadro-síntese dos estudos relacionados aos aplicativos móveis para recrutamento de parceiros. João Pessoa, PB, Brasil, 2018.

\begin{tabular}{|l|l|l|}
\hline \multicolumn{1}{|c|}{$\begin{array}{c}\text { TÍTULO, PERIÓDICO E ANO DE } \\
\text { PUBLICA-ÇÃO }\end{array}$} & \multicolumn{1}{|c|}{ OBJETIVOS } & \multicolumn{1}{c|}{ RESULTADOS } \\
\hline $\begin{array}{l}\text { Gay apps for seeking sex part- } \\
\text { ners in China: Implications for } \\
\begin{array}{l}\text { MSM sexual health. } \\
\text { AIDS Behav, 2015. }\end{array}\end{array}$ & $\begin{array}{l}\text { Examinar dados sociodemográficos e com- } \\
\text { portamento sexual entre Ho-mens que fazem } \\
\text { sexo com homens chineses que usam aplica- } \\
\text { tivos homossexuais em comparação com HSH } \\
\text { que não usam aplicativos gays, a fim de orien- } \\
\text { tar sobre pre-venção, e o risco sexual específi- } \\
\text { co em ambas populações. }\end{array}$ & $\begin{array}{l}\text { Em comparação com os não-usuários de aplicativos, } \\
\text { os usuários de aplicativos eram mais jovens e esta- } \\
\text { vam naixa etária de 26 e 35 anos, possuíam ensino } \\
\text { superior e eram mais informados sobre sexua-lidade. } \\
\text { Tinham uma maior multipli-cidade de parceiros e rea- } \\
\text { lizaram recentemente testes de HIV, quando compa- } \\
\text { rados ao outro grupo. Não houve diferença quanto ao } \\
\text { uso do preservativo entre os dois grupos. }\end{array}$ \\
\hline
\end{tabular}


Virtual Versus Physical Spaces: Which Facilitates Greater HIV Risk Taking Among Men Who HaveSex with Men in Eastand South-EastAsia? AIDS Behavior, 2014.
Investigar se a internet facilita maior risco de HIV entre homens que fazem sexo com homens (HSH) em uma determinada região.
Em comparação com HSH que se reuniu somente com parceiros off-line, aqueles que se reuniu com parceiros online eram menos pro-pensos a ter múltiplos parceiros sexuais masculinos, ter pagado por sexo, ter consumido álcool e outras drogas antes do sexo. HSH que se reuniu com parceiros online e off-line destacou-se como o grupo de maior risco pois eram mais propen-sos a ter múltiplos parceiros, se envolverem em sexo anal desprote-gido e ter consumido álcool antes das relações sexuais.

Os indivíduos que usaram aplicati-vos GSN para buscar parceiros sexuais apresentaram maiores chances de ser positivo para go-norreia e para clamídia comparado a indivíduos que se reuniu com parceiros sem uso de aplicativos. Não houve diferenças significativas na incidência de sífilis e de HIV entre os que conheciam parceiros sem uso de aplicativos, na internet ou através de aplicativos GSN.

Os participantes que foram recruta-dos através de aplicativo eram mais jovens, com maior escolaridade, auto-identificados como brancos e tiveram um maior número de parcei-ros nos últimos 14 dias quando comparados a outros participantes recrutados de outras formas. ment Research.

AIDS Behavior, 2012.

Investigar o uso de aplicativos móveis como ferramenta para o recrutamento de homens que fazem sexo com homens (HSH) para pes-quisas de prevenção do HIV.
Investigar o uso dos aplicativos de smartphone em entrevistas sobre doenças sexualmente transmissí-veis (DST) para encontrar parceiros sexuais.
Alguns indivíduos que usaram apli-cativos para encontrar parceiros tinham em média três parceiros na internet. Parte dos entrevistados tiveram alguma DST e conheciam seu estado sorológico de HIV posi-tivo. Três dos novos casos de DST/HIV estavam entre os parceiros que se encontravam online. Seis parceiros foram localizados usando smartphone e dois foram notifica-dos da sua exposição ao HIV atra-vés de um website.

\section{DISCUSSÃO}

Nesta revisão integrativa, a caracterização sociodemográfica dos usuários de aplicativos móveis, tanto para intervenção em saúde quanto para o recrutamento sexual, mostrou ser majoritariamente jovens, com idade variando entre 16 e 41 anos, que se autodeclararam homem que faz sexo com homem (HSH), brancos, cursando ensino superior, informados sobre sexualidade, e possui média condição social e econômica. É importante considerar que é uma população com diferente perfil quando comparada aos demais segmentos populacionais vulneráveis: baixa escolaridade, pouca informação sobre sexualidade, baixa condição social e econômica ${ }^{(11-13)}$.

Nos últimos anos houve uma mudança na estatística da categoria de exposição ao HIV entre os homens. Em 2014 a exposição heterossexual era a que apresentava maior percentual, e segundo dados do último Boletim Epidemiológico do Ministério da Saúde de 2017, verifica-se que $48,9 \%$ dos casos entre os homens foram decorrentes de exposição homossexual ${ }^{(14)}$.

Quanto ao HIV entre os homens, des- taca-se o aumento na taxa de detecção de 2006 a 2016. Em 2006, a taxa foi de 24,1 casos/100 mil habitantes a qual passou para 25,8 em 2016, representando um aumento de $7,1 \%$. Em jovens de 15 a 19 anos a taxa quase que triplicou e de 20 a 24 anos a taxa mais que duplicou. Ressalta-se que apesar dos aumentos observados nas faixas etárias citadas anteriormente, a maior taxa de detecção em 2016 permaneceu entre os indivíduos na faixa etária de 35 a 39 anos: 49,4 casos/ 100.000 habitantes ${ }^{(14)}$.

Concomitante à mudança no perfil da epidemia nos últimos anos com o aumento 


\section{artigo}

Soares, J.P.; Costas, S.E.J.; Nogueira, W.P.; Souza, L.T.; Lucena, C.K.R.; Pereira, I.L.; Oliveira e Silva, A.C.;

Aplicativos móveis de intervenção como estratégia de prevenção das infecções sexualmente transmissíveis: revisão integrativa

de número de casos de HIV e demais IST entre os jovens, observa-se o surgimento de aplicativos móveis como ferramenta no recrutamento de parceiros sexuais, utilizado principalmente por $\mathrm{HSH}^{(15)}$. Não se pode afirmar que exista uma relação direta do uso dos aplicativos móveis na mudança deste perfil ${ }^{(16)}$, porém pode-se inferir a contribuição deste nas mudanças de comportamento dos jovens quanto as práticas sexuais e de relacionamento. Em estudo desenvolvido em Los Angeles, os jovens que utilizaram aplicativos para reunir parceiros sexuais tiveram maiores chances de ser positivo para gonorreia e clamídia quando comparados a indivíduos que buscavam parceiros em locais tradicionais (bares, saunas, boates, clubes) ou através de outros tipos de redes sociais ${ }^{(17)}$.

A utilização de aplicativos móveis tem se tornado crescente nos últimos anos, a facilidade de identificar parceiros com o perfil desejado, manter o anonimato e facilidade de localização dos mesmos em tempo real, tem motivado muitas pessoas, maioria jovens, a fazer uso desta ferramenta digital. Vários são os aplicativos desenvolvidos com esta finalidade ${ }^{(15,17-19)}$.

A Internet tem atuado cada vez mais na construção de relacionamentos e encontros para sexo casual entre as pessoas. Pessoas novas se conhecem em virtude das redes sociais mais simples, até aplicativos criados especificamente para isso, como os identificados neste estudo.

O uso dos aplicativos digitais favorece uma maior multiplicidade de parcei$\operatorname{ros}^{(15)}$. Estudo desenvolvido em Los Angeles identificou que os jovens que faziam uso destes aplicativos sexuais tiveram um maior número de parceiros nos últimos 14 dias $^{(6)}$. Em Nova York, foi possível observar que os indivíduos que usavam o aplicativo tinham em média três parceiros na Internet ${ }^{(19)}$.

Cumpre assinalar que além do maior número de parceiros, sexo anal desprotegido e consumo de álcool antes das relações sexuais $^{(8,17,19)}$ foram comportamentos de risco presentes neste grupo. Além disso, observou-se maiores taxas de IST/aids entre aqueles indivíduos que se relaciona- ram com parceiros advindos da rede social on-line, quando comparados aos parceiros encontrados em bares, festas, clubes e boates, dentre outros locais tradicionais ${ }^{(17,19)}$.

No entanto, em estudo realizado na Ásia, os pesquisadores concluíram que o ambiente on-line por si só não representa um maior risco para o HIV e demais IST, visto que os $\mathrm{HSH}$ que recrutam parceiros através dos aplicativos estão menos ligados às comunidades gays e espaços físicos, locais esses que aumentam a probabilidade de contato com um maior número de par$\operatorname{ceiros}^{(8)}$.

Por outro lado, apreende-se que os aplicativos móveis podem figurar-se como importantes ferramentas nas estratégias de prevenção as IST/Aids e, consequentemente, à diminuição da vulnerabilidade ${ }^{(16,20)}$. Através do aplicativo comercialmente utilizado nos Estados Unidos, especificamente na Califórnia e em Los Angeles, é possível recrutar indivíduos para estudos relacionados à prevenção do HIV e desenvolver intervenções exitosas. $\mathrm{Na}$ Austrália e na África houve um aumento significativo no uso do preservativo e uma progressão no conhecimento sobre HIV e demais $\operatorname{IST}^{(6,7,21,22)}$.

Em Nova York, os homens que fazem sexo com homens relataram a importância de focar em algumas informações nos aplicativos de intervenção, tais como: testes de HIV, grupos de apoio, uso correto do preservativo, negociação do sexo seguro e reconhecimento dos sinais e sintomas do HIV e outras IST ${ }^{(23)}$.

Ademais, o fato é que estamos vivendo a "geração polegar", indivíduos hiperconectados que recorrem a estas tecnologias móveis em um contexto educacional, o que facilita o acesso rápido, prático e amplo desta geração às informações de saúde.

Neste segmento, observam-se os reais benefícios proporcionados pelo uso dos aplicativos de prevenção, porém, estudos ressaltam a necessidade de uma maior divulgação deste serviço, uma vez que os aplicativos de busca por parceiros são mais evidentes e mais utilizados quando comparados aos de intervenção ${ }^{(20,22,24-25)}$.

Neste estudo, ainda que obtido resul- tados significativos, não foram identificados nas bases de dados pesquisadas artigos realizados no Brasil abordando o uso dos aplicativos como fator de vulnerabilidade e prevenção de IST. Dessa forma, não foi possível fazer um paralelo com a realidade do país.

\section{CONCLUSÃO}

A presente revisão integrativa possibilitou a construção de uma síntese de conhecimento científico acerca dos aplicativos móveis como fator de vulnerabilidade e prevenção de Infecções Sexualmente Transmissíveis. Ressalta-se que parte dos estudos analisados era do tipo transversal, dificultando estabelecer uma relação temporal entre os eventos.

Pôde-se observar que os usuários dos aplicativos móveis eram homens que fazem sexo com homens (HSH), mais jovens, com um maior grau de informação, relaciona-se com um maior número de parceiros em um menor espaço de tempo, bem como possuem uma baixa adesão ao uso do preservativo independente do estado sorológico dos parceiros. Ressalta-se que um maior número de casos de IST/ Aids encontra-se entre os $\mathrm{HSH}$ usuários desta ferramenta digital, quando comparados aos HSH que não faziam o uso, evidenciando a vulnerabilidade.

Por outro lado, essas ferramentas digitais apresentam-se como um grande aliado na prevenção das IST/Aids, uma vez que os aplicativos existentes no mercado foram testados e mostraram-se eficientes, aumentando a adesão ao uso do preservativo e uma progressão no conhecimento sobre as infecções reduzindo a vulnerabilidade. No entanto, se faz necessário uma maior divulgação dos mesmos para que possam ser amplamente utilizados pela população cumprindo com seu papel preventivo. Para serem bem sucedidas, as intervenções através dos aplicativos devem levar em consideração aspectos de privacidade, estigma, normas sociais e diferentes tipos de linguagem.

Infere-se que, apesar dos aplicativos móveis de busca por parceiros serem am- 
plamente utilizados no Brasil, não foram identificados estudos nas bases de dados sobre a temática no país. Fundamenta- do nas afirmações, e pela relevância do tema, evidencia-se a necessidade de um maior investimento em estudos relativos aos aplicativos móveis e sua relação com a vulnerabilidade e prevenção das infecções sexualmente transmissíveis. .

\section{REFERÊNCIAS}

1. Pogetto MRB, Silva MG, Parada CMGL. Prevalence of sexually transmitted diseases in female sex workers in a city in the interior of São Paulo, Brasil. Rev Lat Am Enfermagem. 2011; 19(3):1-7.

2. Sanchez ZM, Nappo SA, Cruz Jl, Carlini EA, Carlini CM, Martins SS. Sexual behavioramong high schoolstudents in Brazil: alcohol consuption and legal and ilegal drug use associated with unprotected sex. Clinics. 2013; 68(4):489-494.

3. World Health Organization (WHO). Sexually Transmitted Infections (STIs). Dec, 2015. [acesso em: 01 set 2018]. Disponivel em: http://who.int/mediacentre/factsheets/fs110/en/.

4. World Health Organization (WHO). Sexually Transmitted Infections (STIs). 2017. [acesso em: 10 out 2018]. Disponível em: http://www.who.int/mediacentre/factsheets/fs110/en/.

5. Penha JC, Aquino CBQ, Neri EAR, Reis TG, Aquino PS, Pinheiro AK. Risk factors for sexually transmitted diseases among sex workers in the interior of Piaui, Brazil. Rev Gaucha Enferm. 2015; 36(2):63-9.

6. Burrel ER, Pines HA, Robbie E, Coleman L, Murphy RD, Hess KL, et al. Use of the location-based social networking rectal microbicide development research. AIDS Behav. 2012; (16):1816-1820

7. Sun CJ, Stowers J, Miller C, Bachmann LH, Rhodes SD. Acceptability and feasibility of using estabilished geosocial and sexual networking mobile applications to promote HIV and STD testing among men who have sex with men. AIDS Behav. 2015; 19(3):543-552.

8. Wei C, Lim SH, Guadamuz TE, Koe S. Virtual versus physicalspaces: with facilitates greater HIV risk talking among men who sex with men in Eastand South EastAsia? AIDS Behav. 2014; (18):1428-1435.

9- Whittemore R. Combining in nursing research: methods and implications. Nurs Res. 2005 Jan-Feb; 54(1):56-62

10. Polit DF, Beck CT. Using research in evidence-based nursing practice. In: Polit DF, Beck CT, editors. Essentials of nursing research: methods, appraisal and utilization. Philadelphia (USA): Lippincott Williams \& Wilkins; 2006. p.457-94.

11. Ursi ES. Prevenção de lesões de pele no perioperatório: revisão integrativa da literatura [Dissertação]. Ribeirão Preto: Universidade de São Paulo, Escola de Enfermagem de Ribeirão Preto, 2005.

12. Granjeiro A, Escuder MML, Castilho EA. Magnitude e Tendência da Epidemia de AIDS em Municípios Brasileiros de 20022006. Rev saúde pública. 2010; 44(3):430-41.

13. Carvalho PMRS, Guimarães RA, Moraes PA, Teles SA, Matos MA Prevalence of signs and symptoms and knowledge about sexually transmitted diseases. Acta Paul Enferm. 2015; 28(1):95-100.

14. Sobrinho-Santos CK, Silva AV, Malheiros AF, Trindade RA, Pagan AA. Relatos de caminhoneiros sobre a prevenção do HIV e o material educacional impresso: reflexões para educação em
saúde.Ciênc. Educ. 2015; 21(4):1011-1030.

15. Ministério da Saúde (Brasil). Boletim Epidemiológico - AIDS e DST. Semanas Epidemiológicas janeiro a junho de 2017. Brasília (DF): Ministério da Saúde, 2017.

16. Bien CH, Best JM, Muessie KE, Wei C, Han L, Tucker JD. Gay apps for seeking sex partners in China: implications for MSM sexual health. AIDS Behav. 2015; 19(6):941-949.

17. Muessig KE, Pike EC, Fowler B, LeGrand S, Parsons JT, Bull SS, et al. Putting prevention in their pockets: developing mobile phone-based HIV interventions for black men who have sex with men. AIDS Patient Care. 2013; 27(4):211-222.

18. Beymer MR, Weiss RE, Bolan RK, Rudy ET, Bourque LB, Rodriguez JP, et al. Sex on demand: geosocial networking phone apps and risk of sexually transmitted infections among a cross-sectional sample of men in Los Angeles Country. Sex Transm Infect. 2014; (90):567-572.

19. Brigmol S, Dourado I. Inquérito sociocomportamental sobre as práticas sexuais desprotegidas entre os homens que fazem sexo com homens usuários de internet. Rev Bras Epidemiol. 2011; 14(3):423-434.

20.Pennise M, Inscho R, Herpin K, Owens Jr J, Bedard BA, Weimer $A C$, et al. Using smartphone apps in STD interviews to find sexual partners. Public Health Reports. 2015; (130):245-252.

21. Holloway IW, Rice E, Gibbs J, Winetrobe H, Dunlap S, Rhoades $\mathrm{H}$. Acceptability os smartphone application-based HIV prevention among Young men who have sex with men. AIDS Behav. 2014; 18(2):285-296.

22. Besoin F, Perez-Navarro A, Cayla JA, Aviño C, Olalla PG. Prevention of sexually transmitted infections using mobile devices and ubiquitous computing. Journal of Health Geographic. 2015; 14(18):1-12.

23. Brennan DJ, Lachowsky NJ, Georgievski G, Rosser BR, MacLachlan D, Murray J, et al. Online outreach services among men who use the internet to seek sex without her men (MISM) in Ontario, Canadá: an online survey. J Med Internet Res. 2015; 17(2):1-13.

24. Aliabadi N, Carballo-Dieguez A, Bakken S, Rojas M, Brown $W$, Carry $M$, et al. Using the Information-Motivation-Behavioral Skills Model to Guide the Development of an HIV Prevention Smartphone Application for High-Risk MSM. AIDS Educ Prev. 2015; 27(6): 522-537.

25.- Czarny HN, Broaddus MR. Acceptability of HIV prevention information delivered through established geosocial networking mobile applications to men who have sex with men. AIDS Behav. 2017; 21(11): 3122-3128.

26. Yue Zhao Y, Zhu X, Pérez AE. MHealth approach to promote Oral HIVself-testing among men who have sex with men in China: a qualitative description. BMC Public Health. 2018; 18(1146): 1-8. 